\title{
miR-3178 inhibits cell proliferation and metastasis by targeting Notch1 in triple- negative breast cancer
}

\author{
Peng Kong ${ }^{1}$, Lie Chen ${ }^{1}$, Muxin Yu', Jing Tao', Jiawei Liu', Yue Wang ${ }^{1}$, Hong Pan', Wenbin Zhou ${ }^{1}$ and Shui Wang ${ }^{1}$
}

\begin{abstract}
Triple-negative breast cancer (TNBC) has a poorer outcome than other subtypes of breast cancer, and the discovery of dysregulated microRNA (miRNA) and their role in tumor progression has provided a new avenue for elucidating the mechanism involved in TNBC. In this study, we identified that miR-3178 was significantly reduced in TNBC, and the low miR-3178 expression correlated with poor overall survival in TNBC but not in non-TNBC. The ectopic overexpression of miR-3178 suppressed TNBC cell proliferation, invasion, and migration by inhibiting the epithelial-to-mesenchymal (EMT) transition. Notch1 was validated as the direct target gene of miR-3178, which was confirmed by the dualluciferase reporter assay. miR-3178 decreased the expression of Notch1 and restoration of Notch1 expression attenuated the inhibitory effects of miR-3178 on cell proliferation, metastasis, and the EMT in TNBC. miR-3178 inhibited cell proliferation and metastasis by targeting Notch1 in TNBC, and the restoration of miR-3178 might be a potential therapeutic strategy for TNBC.
\end{abstract}

\section{Introduction}

Triple-negative breast cancer (TNBC) represents an aggressive subtype of breast cancer characterized by lack of the estrogen receptor, progesterone receptor, and human epidermal growth factor receptor 2 (HER2) ${ }^{1}$. This subtype of patient does not benefit from endocrine and anti-HER2 therapy. Chemotherapy is the common option for adjuvant therapy. TNBC patients have a poorer prognosis than those with other subtypes of breast can$\mathrm{cer}^{2,3}$. This may be partly due to the inherently aggressive clinical behaviors and lack of proper therapeutic targets ${ }^{2,4}$, as well as the high heterogeneity in genomics, epigenomics, transcriptomics, and proteomics that characterize TNBC molecular subtypes ${ }^{5-7}$. For better treatment of TNBC, there is an urgent need to further understand the

Correspondence: Hong Pan (pamghong@163.com) or Wenbin Zhou (zhouwenbin@njmu.edu.cn) or Shui Wang (ws0801@hotmail.com) ${ }^{1}$ Department of Breast Surgery, The First Affiliated Hospital with Nanjing Medical University, 300 Guangzhou Road, 210029 Nanjing, Jiangsu, China These authors contributed equally: Peng Kong and Lie Chen

Edited by E. Candi biological characteristics of this disease and to identify novel therapeutic targets.

The underlying cause of death in majority of breast cancer patients is metastasis, of which the main characteristic is the epithelial-to-mesenchymal transition $(\text { EMT })^{8}$. The EMT acts as the initial event during tumor metastasis ${ }^{9,10}$, leading to loss of cellular adhesion and tumor metastasis ${ }^{8}$. TNBC has a high rate of distant metastasis, and TNBC with mesenchymal features is highly malignant ${ }^{11-13}$. It has been suggested that suppressing the EMT may reduce the metastatic spread of TNBC. Recent studies on EMT focus on molecular levels, and it seems that the EMT does not simply rely on relative genetic alternation $^{14,15}$. The EMT is a highly complex process triggered by diverse extracellular and intracellular signals via EMT-related transcription factors ${ }^{16}$.This may be because EMT is one of multiple steps during tumor metastasis, the purpose of which is to allow cancer cells to leave their primary location. Mesenchymal cancer cells undergo a mesenchymal-to-epithelial transition to form

\section{(c) The Author(s) 2018}

(c) (i) Open Access This article is licensed under a Creative Commons Attribution 4.0 International License, which permits use, sharing, adaptation, distribution and reproduction cc) in any medium or format, as long as you give appropriate credit to the original author(s) and the source, provide a link to the Creative Commons license, and indicate if changes were made. The images or other third party material in this article are included in the article's Creative Commons license, unless indicated otherwise in a credit line to the material. If material is not included in the article's Creative Commons license and your intended use is not permitted by statutory regulation or exceeds the permitted use, you will need to obtain permission directly from the copyright holder. To view a copy of this license, visit http://creativecommons.org/licenses/by/4.0/. 
macro metastatic foci after extravasation in the distant organ.

MicroRNAs (miRNAs) are small noncoding RNAs that are involved in cell proliferation, survival, differentiation, and other essential cellular processes ${ }^{17,18}$. The abnormal expression of miRNAs has been detected in many diseases including cancer. Mounting evidence has reported the dysregulation of miRNAs in TNBC ${ }^{19,20}$. miRNAs are involved in the tumor initiation, progression, diagnostic, prognostic, and therapeutic potential of $\mathrm{TNBC}^{21}$. Moreover, they play important roles in regulating the EMT in breast cancer ${ }^{22}$. The miR-200 family, which inhibits EMT, tumor proliferation, migration, invasion, is downregulated in TNBC ${ }^{22,23}$. Similarly, miR-205, which is downregulated in TNBC, also can reduce proliferation and inhibit $\mathrm{EMT}^{24}$. Thus, regulating miRNA expression may inhibit the EMT, suggesting that using a single miRNA as an anti-cancer agent may provide high therapeutic efficacy without leading to "reactive resistance". However, as an anti-cancer agent, the application of miRNA still has several challenges, such as unsatisfactory delivery efficacy.

In this study, we showed that miR-3178 was downregulated in TNBC. Ectopic overexpression of miR-3178 inhibited the EMT and metastasis in TNBC cells. Importantly, miR-3178 acted as a prognostic factor in TNBC but not in non-TNBC. With the aid of target predictors and a luciferase reporter assay, Notch1 was identified as the target gene of miR-3178. miR-3178 inhibited the expression of Notch1, and restoration of Notch1 expression reversed the inhibitory effects of miR3178 on TNBC cell proliferation, migration, and the EMT. These results indicated that miR-3178 acted as a tumor suppressor in TNBC development by targeting Notch1. Thus, miR-3178 may serve as a powerful anti-cancer therapeutic agent with high efficacy.

\section{Results}

miR-3178 expression was significantly downregulated and acted as a prognostic factor in TNBC but not in non-TNBC

To identify the differential expression of miRNA between TNBC and non-TNBC, miRNA expression microarray analyses were performed using The Cancer Genome Atlas (TCGA) in 1283 breast cancer patients. The miRNA expression levels between 119 TNBC and 1164 non-TNBC patients were compared, and $196 \mathrm{miR}$ NAs (72 upregulated and 124 downregulated) were found to be significantly different between the two groups (Fig. 1a, b). Among these miRNAs, miR-3178 was significantly lower in TNBC compared to the other subtypes (Fig. 1c). To verify the differences in miR-3178 expression, we performed qRT-PCR on 34 patient samples (17 with TNBC and 17 with non-TNBC), and found that miR-3178 was downregulated in TNBC (Fig. 1d). To further determine the prognostic significance of miR-3178, Kaplan-Meier analysis was used. Data on miR-3178 were obtained from 681 breast cancer patients from the TCGA database; 119 had TNBC and 562 had other subtypes (e.g., luminal A, luminal B, and HER-2). High miR-3178 expression contributed to better overall survival (OS) in stage I-IIIB TNBC $(n=66 ; 19$ high expression and 47 low expression) (Fig. 1e). However, high miR-3178 expression was not associated with better OS in non-TNBC (Fig. 1f).

\section{miR-3178 suppressed the cell proliferation and migration of TNBC cells}

miR-3178 mimic lentivirus was transfected into two TNBC cell lines (MDA-MB-231 and SUM-1315), and the transfection efficiency was determined by qRT-PCR (Fig. 2a). The CCK-8 assay showed that the proliferation rate of TNBC cells transfected with miR-3178 mimics was significantly inhibited compared with the negative control group (NC) (Fig. 2b). The mRNA expression of the cell proliferation-associated marker Ki-67 and the cell cyclerelated genes cyclinD1 and cyclin E1 was significantly reduced by miR-3178 mimics (Fig. 2c). Furthermore, the colony formation assay revealed that the colony number of TNBC cells transfected with miR-3178 mimics was significantly reduced compared with those transfected with NC (Fig. 2d). The wound healing assay and transwell invasion assay were performed to detect the effects of miR-3178 on TNBC migration and invasion (Fig. 2e, f). The results of the wound healing assay showed that miR3178 mimics decreased TNBC cell migration; similar results were detected with the transwell invasion assay. The migratory and invasive abilities of TNBC cells transfected with miR-3178 mimics also decreased.

Further, miR-3178 inhibitor was transfected into SUM1315 and MDA-MB-231 cells, and inhibited the expression of miR-3178 (Fig. S1A). The CCK-8 assay, wound healing assay and transwell invasion assay showed that suppression of miR-3178 can increase TNBC cell proliferation, migration and invasion, respectively (Fig S1BD).

These results illustrated that miR-3178 inhibited the malignant characteristics of TNBC cells.

\section{miR-3178 inhibited the EMT in TNBC cells}

To determine the effects of miR-3178 on the EMT, the EMT markers were detected by qRT-PCR and western blotting. As shown in Fig. 3a, b, the epithelial cell marker of the EMT, E-cadherin, was significantly upregulated, but mesenchymal markers such as vimentin and N-cadherin were markedly downregulated in SUM-1315 and MDAMB-231 cells transfected with miR-3178 mimics. 


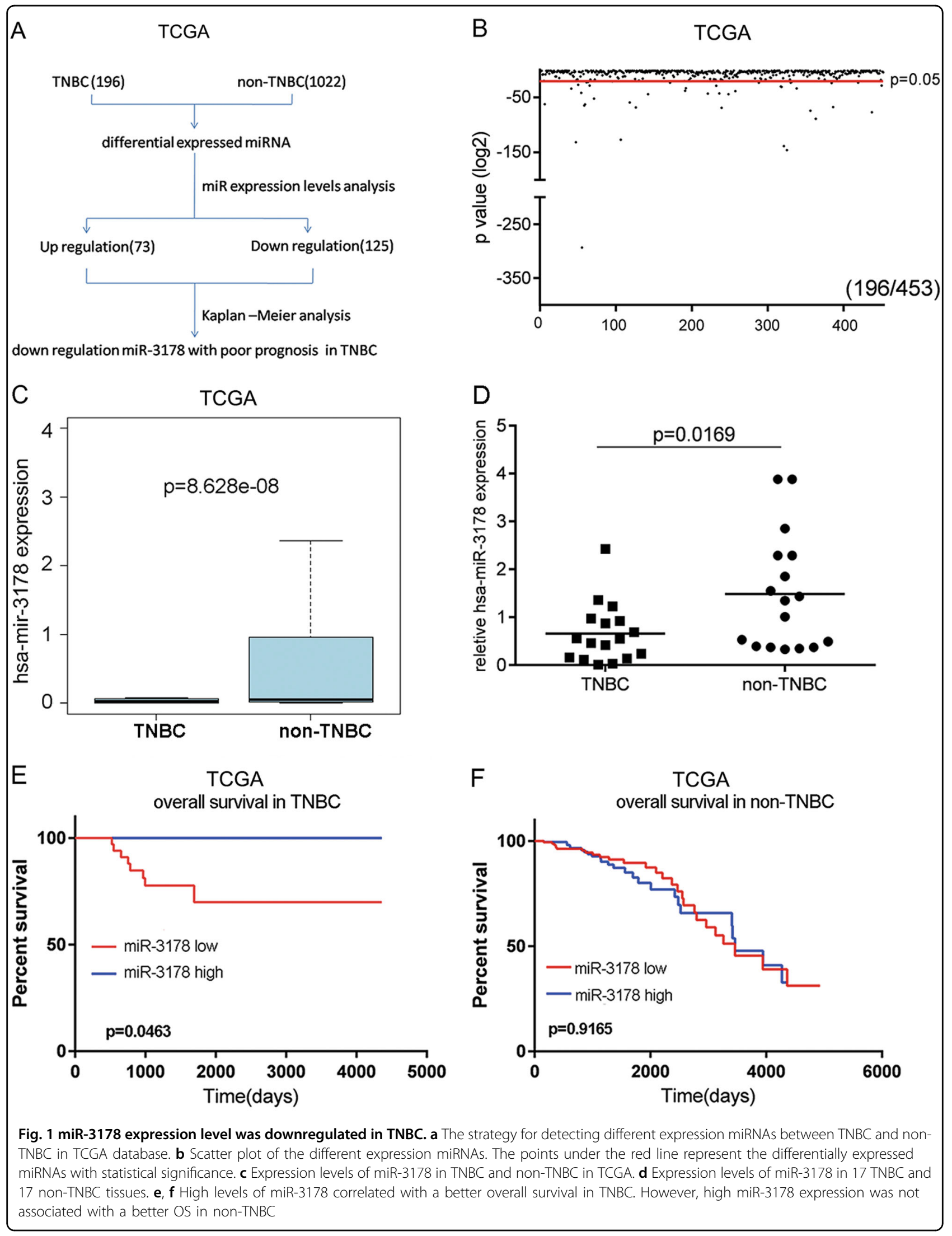




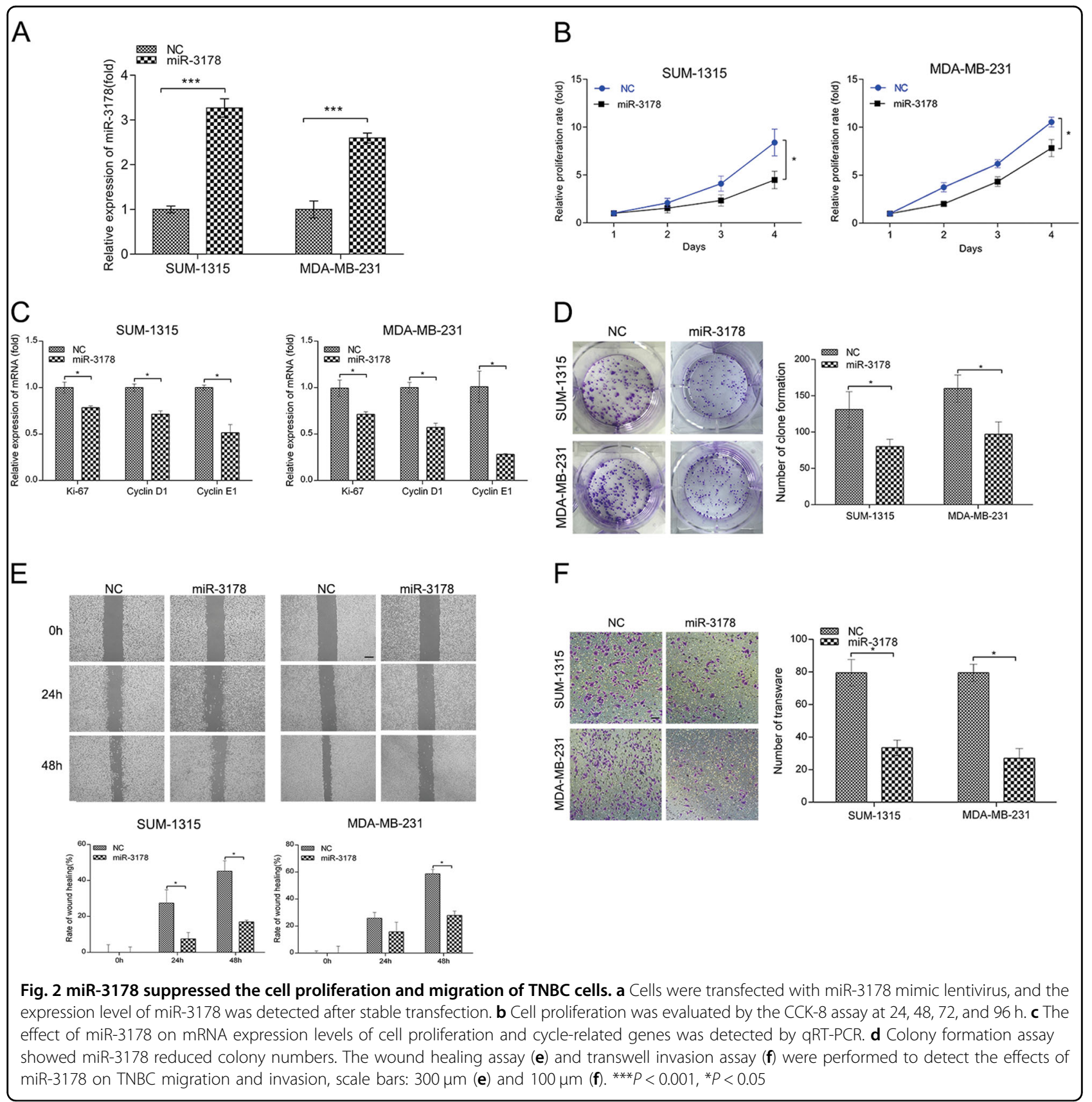

Furthermore, miR-3178 also downregulated the mRNA and protein expression of Snail and Slug, transcription factors that directly regulate the EMT (Fig. 3c, d). This further supports the hypothesis that miR-3178 reduces tumor malignancy by inhibiting the EMT in TNBC.

\section{Notch1 was a direct downstream target of miR-3178}

The miR data bases, TargetScan and miRNATar Base were used to predict the target genes of miR-3178 that may regulate tumor growth and metastasis, and Notch1 was recognized as a potential target. Then, the luciferase assay was performed to determine whether there was a direct correlation between miR-3178 and Notch1. We constructed luciferase reporter constructs that had either a WT-notch1-3'-UTR or mut-notch1-3'-UTR sequence of the miR-3178-binding site (Fig. 4a). The relative luciferase activity was decreased after co-transfection with miR-3178 and WT-notch1-3'-UTR in MDA-MB-231 and SUM-1315 cells. The decreased luciferase activity was not checked after transfected miR-3178 with mut-notch1-3'UTR (Fig. 4b). To further support the specific regulation of Notch 1 by miR-3178, we measured Notch1 expression 


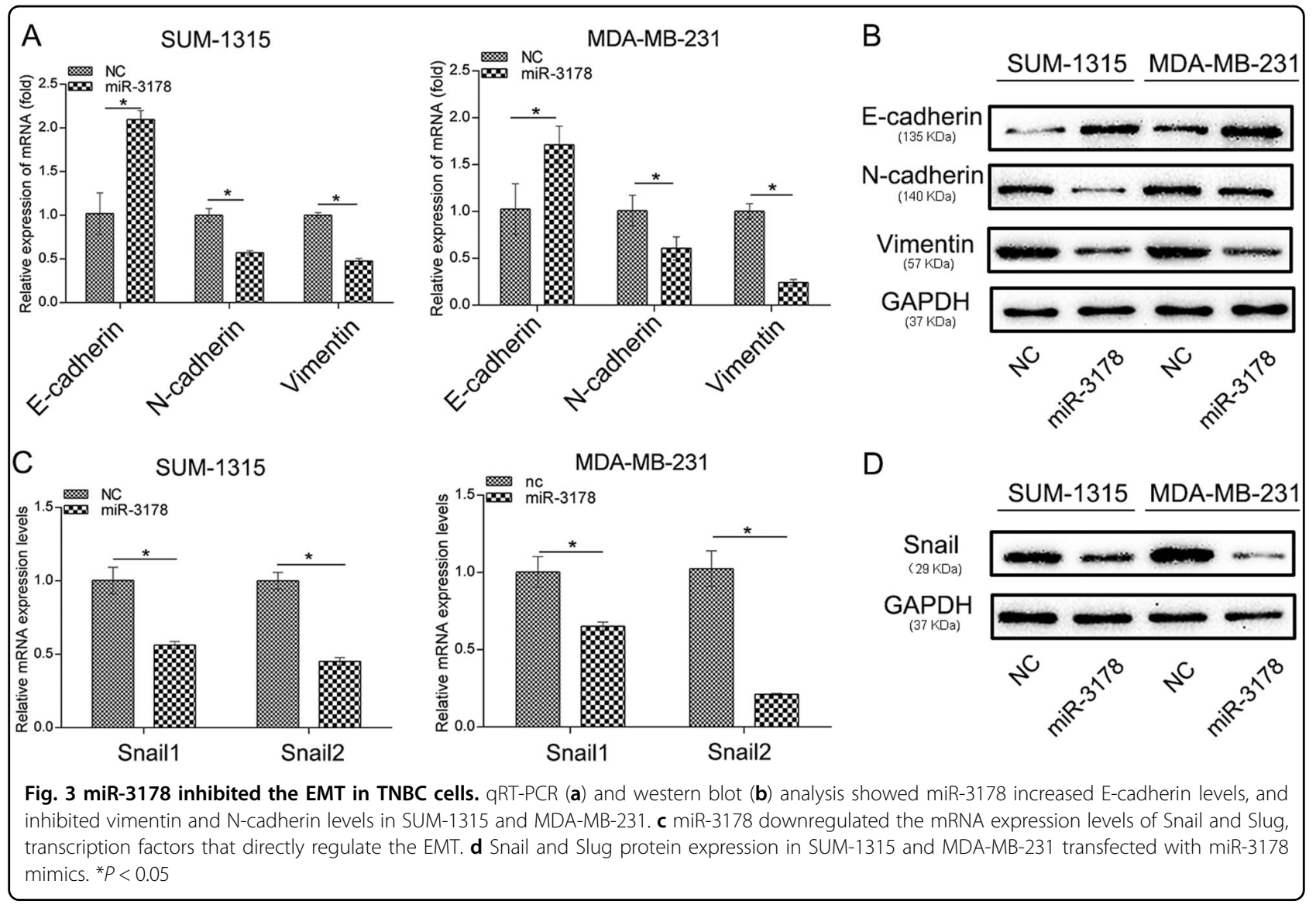

in miR-3178 mimic-transfected cells by qRT-PCR and western blot analysis. The overexpression of miR-3178 decreased Notch1 mRNA and protein expression, and NF- $\mathrm{B}$ (a downstream factor of Notch1) was also inhibited by miR-3178 (Fig. 4c, d).

\section{Restored expression of Notch1 attenuated inhibition of cancer cell proliferation by miR-3178 in TNBC cells}

si-Notch1 was transfected into SUM-1315 and MDAMB-231 cells to confirm the effects of miR-3178 on proliferation, migration and invasion in TNBC were mediated by downregulation of Notch1. Then we utilized the qRT-PCR and western blot to analysis the downexpression of Notch1 (Fig. S2A, B). The CCK-8 assay, wound healing assay and transwell invasion assay were also performed, and we found that inhibition of Notch1 could suppress the proliferation, migration, and invasion in SUM-1315 and MDA-MB-231 cells (Fig. S2C-E).

To further investigate whether miR-3178 exerts its antitumor effects by targeting Notch1, Notch1 expression was upregulated by Notch1 plasmid (p-Notch1). As shown in Fig. 5a, b, Notch1 and NF-kB were downregulated by miR3178 mimics in SUM-1315 and MDA-MB-231 cells, which was reversed with transfection of p-Notch1. Fig. 5c shows that the inhibition of cell proliferation by miR-3178 was also reversed by p-Notch1. Furthermore, p-Notch1 restored the mRNA levels of Ki-67, cyclinD1, and cyclin E1, which were inhibited by miR-3178 mimics (Fig. 5d). In addition, p-Notch1 reversed the inhibitory effects of miR3178 on colony formation (Fig. 5e).

\section{miR-3178 regulated Notch1-mediated migration and the} EMT through Snail1 in TNBC cells

Studies have shown that Notch1 is an important regulator of the EMT and is associated with tumor migration and invasion in breast cancer. As shown in Fig. 6a, b, restoring the expression of Notch1 by p-Notch1 attenuated the inhibition of miR-3178 on cell migration and invasion. To further confirm that miR-3178 regulated the EMT by targeting Notch1, the Notch1 downstream factor Snail1 was detected by qRT-PCR and western blot analysis. After increasing the activity of Notch 1 by p-Notch1, the expression of Snail1 was upregulated compared to expression in miR-3178 mimic-transfected cells. Expression of the EMT markers affected by miR-3178 mimics was also reversed by p-Notch1 (Fig. 6c, d). 




miR-3178 inhibited tumor growth in a nude mouse model of TNBC

To assess the effects of miR-3178 on tumor growth in vivo, miR-3178, miR-3178/p-Notch1, miR-3178/p-NC, and $\mathrm{m}-\mathrm{NC}$ MDA-MB-231 and SUM-1315 cells were subcutaneously implanted into nude mice. Tumor volume and weight were recorded. The tumor weight of miR-3178 and miR-3178/p-NC was significantly lower than that with $\mathrm{m}-\mathrm{NC}$ and miR-3178/p-Notch1 (Fig. 7a). Same as the cell proliferation observed in vitro, miR-3178 significantly decreased tumor volume compared with m-NC. miR-3178/p-Notch1 partly neutralized the inhibitory effects of miR-3178 on tumor volume (Fig. 7b).

\section{Discussion}

Due to a lack of treatment targets, chemotherapy has remained the main therapeutic strategy for TNBC. TNBC has high pathologic complete response rates after neoadjuvant chemotherapy ${ }^{25,26}$, but it also has more chemoresistance and a worse prognosis than other breast cancer subtypes ${ }^{2,3,27}$. Thus, it is necessary to find novel targets for TNBC. The current possible targets for TNBC are mainly aberrant signal transduction pathways or overexpressed proteins in $\mathrm{TNBC}^{28-33}$, such as mTOR inhibitors, Src tyrosine kinase inhibitors, PARP inhibitors, anti-EGFR, and anti-VEGFR therapies. The abnormal expression of miRNAs correlates with tumor initiation and progression in $\mathrm{TNBC}^{21}$. The reason of miRNAs reduction may be relative promoter hypermethylation in $\mathrm{TNBC}^{34}$. Besides, Long noncoding RNAs (LncRNAs) could inhibit miRNAs directly in TNBC ${ }^{35}$. miRNAs, small noncoding RNAs, bind to the $3^{\prime} \mathrm{UTR}$ on target mRNAs, leading to mRNA degradation and inhibition of protein translation. A single miRNA can regulate multiple target genes, which makes miRNAs large and powerful regulators of tumor genesis and progression ${ }^{36}$. In our study, miR-3178 was downregulated in TNBC compared with other subtypes of breast cancer. The ectopic overexpression of miR-3178 significantly reduced the proliferation and migration of MDA-MB-231 and SUM-1315 TNBC cells, and similar results were observed in the animal models. These results demonstrate the anti-tumor characteristics of miR-3178 in TNBC.

To illustrate the inhibition of miR-3178 in TNBC, the underlying mechanisms were analyzed. The miRNAs exhibited anti-tumor effects mainly through suppressing proliferation and migration and promoting apoptosis in $\mathrm{TNBC}^{37-39}$. TNBC has more distant metastasis than other subtypes of breast cancer, and several studies have confirmed that metastasis is more likely to occur in TNBC with mesenchymal characteristics ${ }^{13,40}$. Thus, reversing the EMT may be a possible strategy for treating TNBC. The 


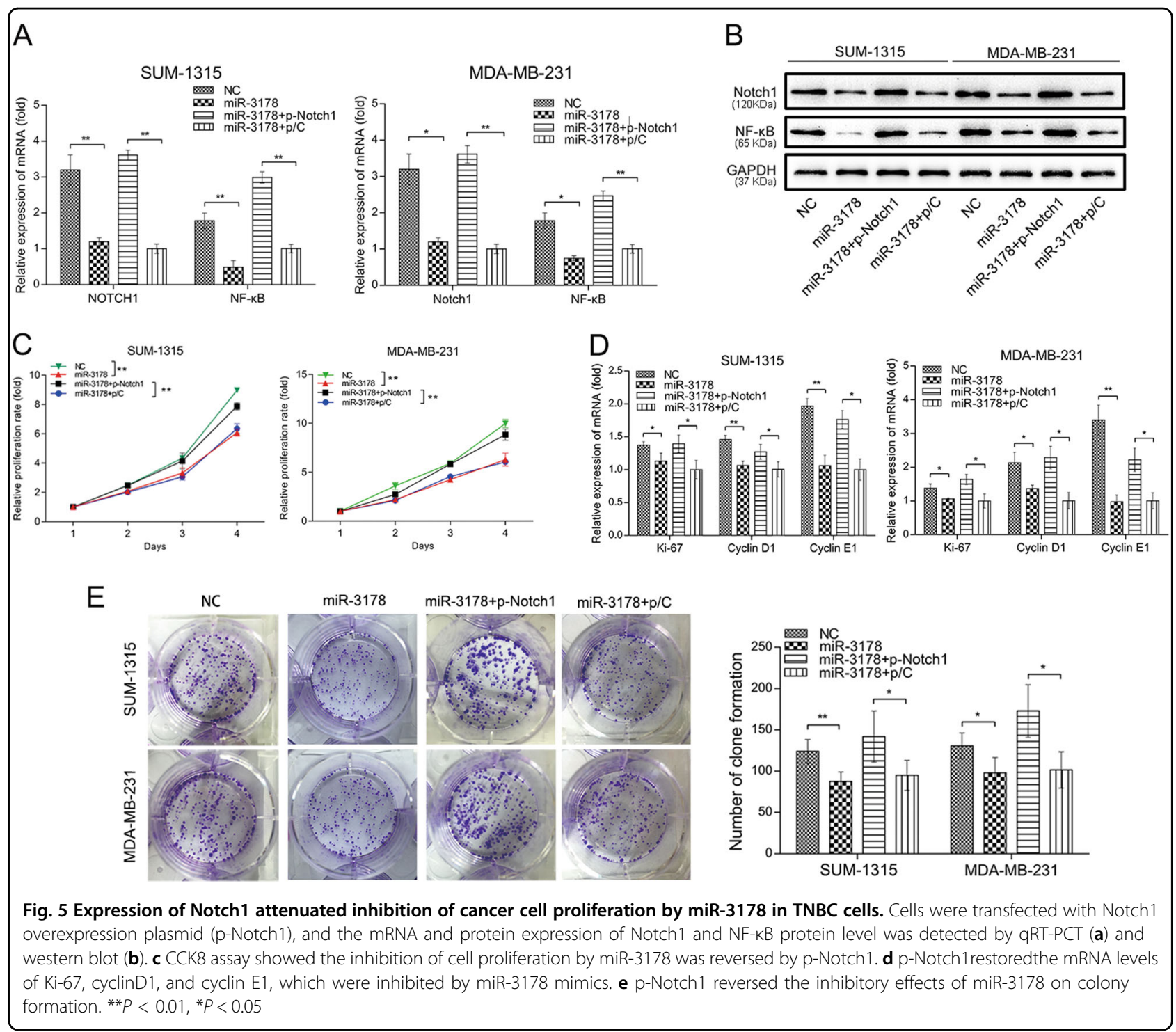

EMT is a fundamental mechanism for embryo development, wound healing, and organ fibrosis, and is also the initial event of tumor metastasis. The EMT is a highly complex process that is regulated by the extracellular matrix, autocrine, or paracrine cell factors ${ }^{16}$, as well as by miRNAs ${ }^{41}$, which are associated with the EMT and metastasis of breast cancer ${ }^{19,42}$. Our data indicated that mesenchymal characteristics were reduced after the upregulation of miR-3178 in TNBC cells. Analysis of the database showed that Notch1 was a potential target gene for miR-3178. Furthermore, miR-3178 directly combined with Notch1 and inhibited its expression. This further supports the hypothesis that miR-3178 inhibits the EMT by targeting Notch 1 in TNBC.

The Notch family is important for the intercellular communication system, and is involved in cell differentiation, proliferation, death, and cancer initiation ${ }^{43,44}$.
In solid tumors, Notch signaling regulates tumor growth, cell survival, angiogenesis, cell differentiation, and development. Besides, Notch signaling plays an important role in the development of cancer stem cells $(\mathrm{CSCs})^{45}$, and maintains CSCs stemness, self-renewal and

proliferation $^{46,47}$. The abnormal expression of Notch has also been detected in $\mathrm{TNBC}^{48,49}$, and a phase I trial of RO4929097 (notch/secretase inhibitor) is currently investigating Notch1 in combination with neoadjuvant chemotherapy (paclitaxel and carboplatin) for inoperable TNBC. Notch1, one of four Notch receptors, is overexpressed in $\mathrm{TNBC}^{48}$, indicating that it could be a therapeutic target in TNBC. In our study, miR-3178 significantly reduced the expression of Notch1, and the restored expression of Notch1 attenuated the inhibition of miR-3178 on tumor proliferation and metastasis. 




In conclusion, the results of this study showed that miR3178 was downregulated and acted as a tumor suppressor by reducing cell growth and metastasis in TNBC. Importantly, miR-3178 acted as a prognostic factor in TNBC but not in non-TNBC. These anti-tumor effects of miR-3178 were dependent on its direct regulation of Notch1. These data may provide a promising approach for the treatment of TNBC.

\section{Methods}

\section{Cell culture and animals}

Two human TNBC cell lines were used in this study: MDA-MB-231 was obtained from America Type Culture Center (ATCC, Manassas, VA, USA), and SUM-1315 was kindly provided by Stephen Ethier (University of
Michigan, Ann Arbor, MI, USA). The cells were cultured in Dulbecco's modified Eagle medium (DMEM, GIBCO, Suzhou, China) containing 10\% fetal bovine serum and 1\% penicillin-streptomycin, and maintained at $37^{\circ} \mathrm{C}$ with $5 \%$ $\mathrm{CO}_{2}$.

\section{Patient tissue samples}

The breast cancer tissues and adjacent normal tissues were obtained from the First Affiliated Hospital with Nanjing Medical University. The breast cancer patients did not receive any chemotherapy or endocrine therapy before tumor resection. All tissues were frozen in liquid nitrogen immediately and stored at $-80^{\circ} \mathrm{C}$ after surgical resection. Informed consent was obtained from each patient, and this study was approved by the Ethics 


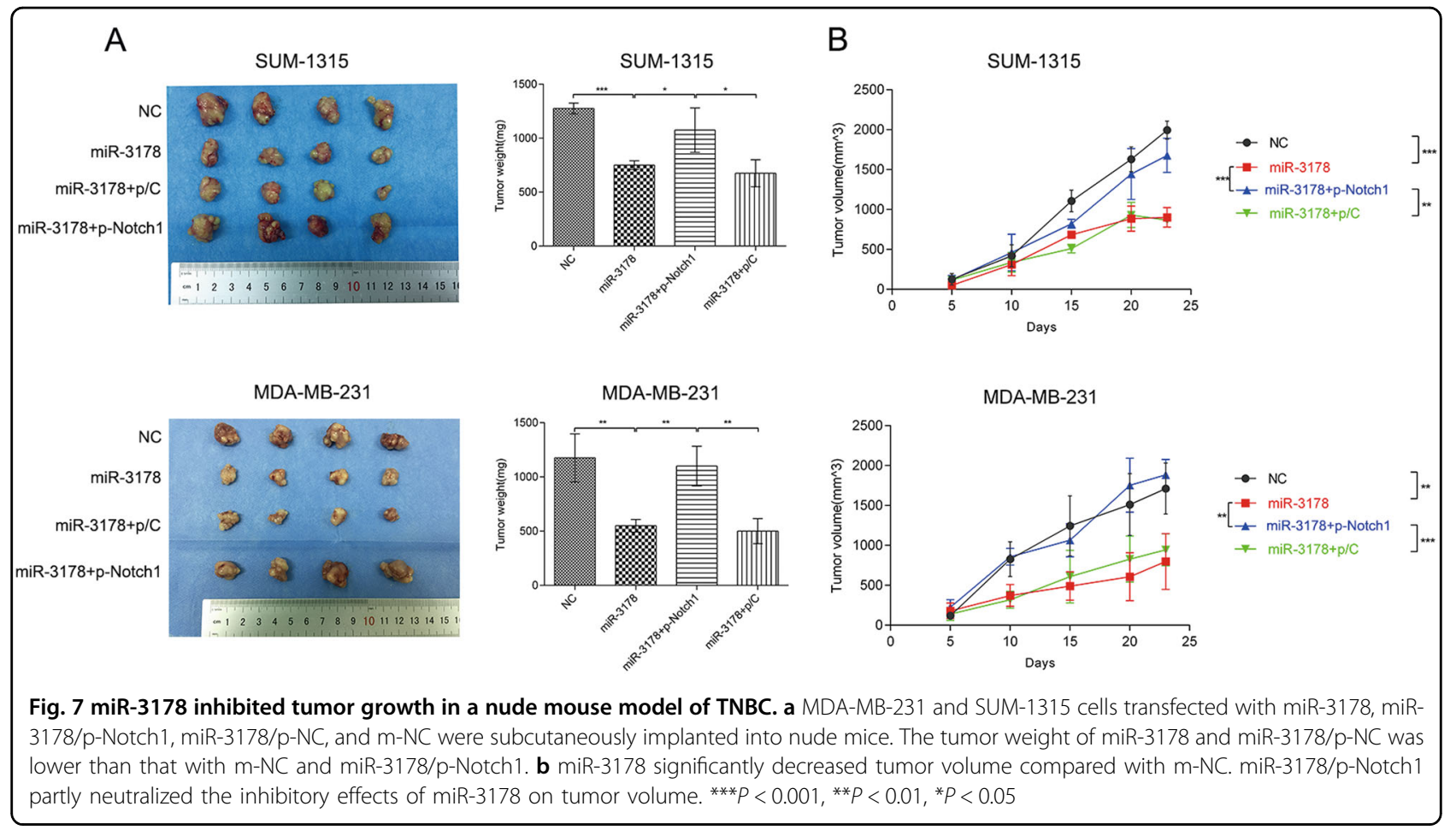

Committees of the First Hospital Affiliated Hospital with Nanjing Medical University.

\section{Transfection of lentivial vectors in vitro}

Cells in which miR-3178 was overexpressed were generated by transfecting miR-3178 mimics. A total of $2 \times 10^{5}$ breast cancer cells/well in 6-well plates were grown overnight and transduced by adding $8 \mu \mathrm{g} / \mathrm{mL}$ polybrene and $10 \mu \mathrm{L}$ lenti-miR-3178 and lenti-miR-Scramble lentiviral particles (LV5-EF1a-GFP/Puro; Shanghai GenePharma Co., Shanghai, China). After $24 \mathrm{~h}$, stably transfected cells were selected using $0.3 \mu \mathrm{g} / \mathrm{mL}$ puromycin for 4 weeks.

\section{Animal models}

Animal experiments were approved by the Institutional Animal Care and Use Committee, Nanjing Medical University (Nanjing, China). Female Balb/c nude mice (4-6 weeks old) were purchased from the Model Animal Research Center of Nanjing University and maintained under specific pathogen-free conditions in the Animal Core Facility of Nanjing Medical University. A total of $2 \times$ $10^{6}$ cells were injected on the left side of the second pair of mammary fat pads of nude mice. Tumor volume was detected every 5 days and calculated as follows: total tumor volume $\left(\mathrm{mm}^{3}\right)=$ length $\times$ width $^{2} / 2$. Weight loss was monitored every 5 days. Mice were sacrificed when their weight decreased by $30 \%$. At 25 days after injection, the tumor specimens were removed, weighed, and fixed in $10 \%$ buffered formalin for further analysis.

\section{Cell proliferation, colony formation, migration, and invasion assay in vitro}

A total of $3 \times 10^{3} /$ well cells were seeded in 96-well plates, and cell proliferation was detected using Cell Counting Kit-8 (CCK-8; Dojindo Laboratories, Kumamoto, Japan) according to the manufacturer's instructions. To evaluate colony formation, the colony formation assay was performed. Briefly, 500 cells per well were seeded into 6-well plates and incubated for about 2 weeks. Then, the plates were washed three times with phosphatebuffered saline and stained with crystal violet (Beyotime, Shanghai, China). The number of cell colonies formed per well was counted. For the cell migration assay, the wound healing test was performed. To this end, 3 lines of $1 \mathrm{~cm}$ intervals were drawn behind 6 -well plates. Then, $6 \times 10^{5}$ transfected cells were inoculated into the 6-well plates and incubated for $24 \mathrm{~h}$. For the scratch test, when the cell density reached $80-90 \%$, scratching the cells with the lines perpendicular to the previously painted line. Three fields were randomly selected from each well, and cell movements in the scratch were observed and photographed at 0,24 , and $48 \mathrm{~h}$. The width of the scratch was detected with Image Pro 6.0 software. These experiments 
Table 1 The sequence of primer

\begin{tabular}{|c|c|}
\hline Primer & Sequence \\
\hline \multicolumn{2}{|l|}{ GAPDH } \\
\hline Fwd & 5'-GAAGGTGAAGGTCGGAGTC-3' \\
\hline Rev & 5'- GAAGATGGTGATGGGATTTC-3' \\
\hline \multicolumn{2}{|l|}{ Ki-67 } \\
\hline Fwd & 5'-ACGCCTGGTTACTATCAAAAGG-3' \\
\hline Rev & 5'- CAGACCCATTTACTTGTGTTGGA-3' \\
\hline \multicolumn{2}{|c|}{ Cyclin D1 } \\
\hline Fwd & 5'-GCTGCGAAGTGGAAACCATC-3' \\
\hline Rev & 5'-CCTCCTTCTGCACACATTTGAA-3' \\
\hline \multicolumn{2}{|c|}{ Cyclin E1 } \\
\hline Fwd & 5'-ACTCAACGTGCAAGCCTCG-3' \\
\hline Rev & 5'-GCTCAAGAAAGTGCTGATCCC-3' \\
\hline \multicolumn{2}{|l|}{$\mathrm{CDH} 1$} \\
\hline Fwd & 5'- CAGCACGTACACAGCCCTAA-3' \\
\hline Rev & 5'- TGAGGCTTTGGATTCCTCTC-3' \\
\hline \multicolumn{2}{|l|}{$\mathrm{CDH} 2$} \\
\hline Fwd & 5'- CAGCACGTACACAGCCCTAA-3' \\
\hline Rev & 5'- TGAGGCTTTGGATTCCTCTC-3' \\
\hline \multicolumn{2}{|c|}{ vimentin } \\
\hline Fwd & 5'- CAGATGCGTGAAATGGAAGA-3' \\
\hline Rev & 5'- CTCAATGTCAAGGGCCATCT-3' \\
\hline \multicolumn{2}{|l|}{ Snail1 } \\
\hline Fwd & 5'-CCTCCACGAGGTGTGACTAACT-3' \\
\hline $\operatorname{Rev}$ & 5'-CCGACAAGTGACAGCCATTA -3' \\
\hline \multicolumn{2}{|l|}{ Snail2 } \\
\hline Fwd & 5'-CGCAATCAATGTTTACTCGAAC-3' \\
\hline Rev & 5'- TCTCAATCTAGCCATCAGCAAA-3' \\
\hline \multicolumn{2}{|l|}{ Notch1 } \\
\hline Fwd & 5'-ATCGACATGGCCGAATGGAA-3' \\
\hline Rev & 5'-ATGATGTCCACGCCCTTCTG-3' \\
\hline \multicolumn{2}{|l|}{$N F-k B$} \\
\hline Fwd & 5'-GGTGCGGCTCATGTTTACAG-3' \\
\hline $\operatorname{Rev}$ & 5'-GATGGCGTCTGATACCACGG-3' \\
\hline \multicolumn{2}{|c|}{ miR-3178 } \\
\hline Fwd & 5'-GGGGCGCGGCCGGATCG-3 \\
\hline Rev & 5'-GCTGTCAACGATACGCTACGTAACG-3' \\
\hline \multicolumn{2}{|l|}{ U6 } \\
\hline Fwd & 5'-CTCGCTTCGGCAGCACA-3' \\
\hline Rev & 5'-AACGCTTCACGAATTTGCGT-3' \\
\hline
\end{tabular}

were repeated at least three times. The transwell assays (Minicell, Millipore, USA) was used to quantify cell invasion. The transwell membranes were pre-coated with $50 \mu \mathrm{L}$ matrigel (Corning Inc., Corning, NY, USA), and $200 \mu \mathrm{L}$ cell suspension $\left(5 \times 10^{3}\right.$ cells $)$ in serum-free DMEM was added to the upper chamber with $8.0 \mu \mathrm{m}$ pore size membrane inserts in the 24-well plates. Cells that migrated to the outside of the membrane were stained with crystal violet (Beyotime) after $24 \mathrm{~h}$. The cell number was counted at a $\times 200$ magnification in five randomly selected regions per well.

\section{Luciferase reporter assay}

Possible miR-3178 binding sites were obtained from a miRNA database (targetscan.org). Wild-type Notch1 (WT-notch1-3'-UTR) and mutant Notch1 (mut-notch13'-UTR) were purchased from Shanghai Genechem Co., Ltd. (Shanghai, China). For the luciferase reporter assay, cells were co-transfected with WT-notch1-3'-UTR or mut-notch1-3'-UTR plasmids and miR-3178. Firefly luciferase activities were measured using the Dual Luciferase Assay System (Promega, Madison, WI, USA) $48 \mathrm{~h}$ after transfection, and the results were normalized with Renilla luciferase.

\section{Quantitative real-time PCR}

Total mRNA was extracted using TRIzol reagent (Invitrogen, Carlsbad, CA, USA). Quantitative real-time PCR (qRT-PCR) for miRNA detection was performed with the indicated TaqMan MicroRNA Assay (Thermo Fisher Scientific, Waltham, MA, USA) according to manufacturer's instructions, and normalized to U6 RNA levels. Reverse transcription of total RNA was performed using an RT-PCR Kit (TaKaRa, Otsu, Japan), according to previously described procedures ${ }^{50}$. The primer sequences used for detecting target genes are shown in Table 1.

\section{Western blot analysis}

Western blot analysis was performed as previously described $^{51}$. The antibodies used were as follows: antiE-cadherin (Cell Signaling Technology [CST], Danvers, MA, USA), anti-N-cadherin (CST), anti-Snail (CST), anti-NF- $\mathrm{kB}$ (CST), anti-vimentin (Abcam, Cambridge, MA, USA), anti-Notch1 (Abcam) and anti-GAPDH (Beyotime).

\section{Statistics}

Numerical data are presented as mean \pm standard deviation. All of the data were statistically analyzed by the Student's $t$-test or one-way analysis of variance. IBM SPSS Statistics version 22 (Chicago, IL, USA) was used, and $p<0.05$ was considered statistically significant. 


\section{Acknowledgements}

We thank Dr. Stephen Ethier for providing breast cancer cells and Dr. Jifu Wei for the scientific editing of the manuscript. Funding This work was supported in part by the National Natural Science Foundation of China (81572607, $81572595,81572602,81502299,81502286,81702617$, and 81771953), and a project Funded by the Priority Academic Program Development of Jiangsu Higher Education Institutions.

\section{Authors contributions}

S.W. has contributed to the conception and design of the study, the analysis and interpretation of data, the revision of the article as well as final approval of the version to be submitted. W.Z. and H.P. participated in the design of the study, performed the statistical analysis, and drafted and revised the article. P. K., L.C., M.Y., J.T., J.L., and Y.W. performed the experimental study. All authors read and approved the final version of the manuscript.

\section{Conflict of interest}

The authors declare that they have no conflict of interest.

\section{Publisher's note}

Springer Nature remains neutral with regard to jurisdictional claims in published maps and institutional affiliations.

Supplementary Information accompanies this paper at (https://doi.org/ 10.1038/s41419-018-1091-y).

Received: 19 May 2018 Revised: 3 September 2018 Accepted: 10 September 2018

Published online: 17 October 2018

\section{References}

1. Gluz, O. et al. Triple-negative breast cancer - current status and future directions. Ann. Oncol. 20, 1913-1927 (2009).

2. Dent, R. et al. Triple-negative breast cancer: clinical features and patterns of recurrence. Clin. Cancer Res 13, 4429-4434 (2007).

3. Malorni, L. et al. Clinical and biologic features of triple-negative breast cancers in a large cohort of patients with long-term follow-up. Breast Cancer Res. Treat. 136, 795-804 (2012)

4. Weigelt, B. \& Reis-Filho, J. S. Histological and molecular types of breast cancer: is there a unifying taxonomy? Nat. Rev. Clin. Oncol. 6, 718-730 (2009).

5. Banerji, S. et al. Sequence analysis of mutations and translocations across breast cancer subtypes. Nature 486, 405-409 (2012).

6. Shah, S. P. et al. The clonal and mutational evolution spectrum of primary triple-negative breast cancers. Nature 486, 395-399 (2012).

7. Curtis, $C$. et al. The genomic and transcriptomic architecture of 2,000 breast tumours reveals novel subgroups. Nature 486, 346-352 (2012).

8. Felipe, L. J., Nofech-Mozes, S., Bayani, J. \& Bartlett, J. M. EMT in Breast Carcinoma-A Review. J Clin Med 5, 65 (2016).

9. Sleeman, J. P. The lymph node as a bridgehead in the metastatic dissemination of tumors. Recent Results Cancer Res. 157, 55-81 (2000).

10. Brabletz, T., Kalluri, R., Nieto, M. A. \& Weinberg, R. A. EMT in cancer. Nat. Rev. Cancer 18, 128-134 (2018).

11. Yang, Y. et al. DEK promoted EMT and angiogenesis through regulating PI3K $\mathrm{AKT} / \mathrm{mTOR}$ pathway in triple-negative breast cancer. Oncotarget $\mathbf{8}$, 98708-98722 (2017).

12. Pitner, M. K., Taliaferro, J. M., Dalby, K. N. \& Bartholomeusz, C. MELK: a potential novel therapeutic target for TNBC and other aggressive malignancies. Expert Opin. Ther. Targets 21, 849-859 (2017).

13. Luo, C. W. et al. CHD4-mediated loss of E-cadherin determines metastatic ability in triple-negative breast cancer cells. Exp. Cell Res. 363, 65-72 (2018).

14. Lamouille, S., Xu, J. \& Derynck, R. Molecular mechanisms of epithelialmesenchymal transition. Nat. Rev. Mol. Cell Biol. 15, 178-196 (2014).

15. Bill, R. \& Christofori, G. The relevance of EMT in breast cancer metastasis: correlation or causality? FEBS Lett. 589, 1577-1587 (2015).
16. Thiery, J. P. \& Sleeman, J. P. Complex networks orchestrate epithelialmesenchymal transitions. Nat. Rev. Mol. Cell Biol. 7, 131-142 (2006).

17. Sayed, D. \& Abdellatif, M. MicroRNAs in development and disease. Physiol. Rev. 91, 827-887 (2011)

18. Ambros, V. The functions of animal microRNAs. Nature 431, 350-355 (2004).

19. Goh, J. N. et al. microRNAs in breast cancer: regulatory roles governing the hallmarks of cancer. Biol. Rev. Camb. Philos. Soc. 91, 409-428 (2016).

20. Yang, F., Zhang, W., Shen, Y. \& Guan, X. Identification of dysregulated microRNAs in triple-negative breast cancer (review). Int. J. Oncol. 46, 927-932 (2015).

21. Sui, $X$. et al. MicroRNAs-mediated cell fate in triple negative breast cancers. Cancer Lett. 361, 8-12 (2015).

22. Bottai, G. et al. Integrated MicroRNA-mRNA profiling identifies oncostatin M as a marker of mesenchymal-like ER-negative/HER2-negative breast cancer.Int. J. Mol. Sci. 18, 194 (2017).

23. D'Ippolito, E. et al. miR-9 and miR-200 Regulate PDGFRbeta-mediated endothelial differentiation of tumor cells in triple-negative breast cancer. Cancer Res 76, 5562-5572 (2016).

24. Piasecka, D., Braun, M., Kordek, R., Sadej, R. \& Romanska, H. MicroRNAs in regulation of triple-negative breast cancer progression. J. Cancer Res. Clin. Oncol. 144, 1401-1411 (2018).

25. von Minckwitz, G. et al. Definition and impact of pathologic complete response on prognosis after neoadjuvant chemotherapy in various intrinsic breast cancer subtypes. J. Clin. Oncol. 30, 1796-1804 (2012).

26. Liedtke, $\mathrm{C}$. et al. Response to neoadjuvant therapy and long-term survival in patients with triple-negative breast cancer. J. Clin. Oncol. 26, 1275-1281 (2008).

27. Carey, L. A. et al. The triple negative paradox: primary tumor chemosensitivity of breast cancer subtypes. Clin. Cancer Res. 13, 2329-2334 (2007).

28. Robert, N. J. et al. RIBBON-1: randomized, double-blind, placebo-controlled, phase III trial of chemotherapy with or without bevacizumab for first-line treatment of human epidermal growth factor receptor 2-negative, locally recurrent or metastatic breast cancer. J. Clin. Oncol. 29, 1252-1260 (2011).

29. Burstein, $H$. J. et al. Phase II study of sunitinib malate, an oral multitargeted tyrosine kinase inhibitor, in patients with metastatic breast cancer previously treated with an anthracycline and a taxane. J. Clin. Oncol. 26, 1810-1816 (2008).

30. Corkery, B., Crown, J., Clynes, M. \& O'Donovan, N. Epidermal growth factor receptor as a potential therapeutic target in triple-negative breast cancer. Ann. Oncol. 20, 862-867 (2009).

31. Hastak, K., Alli, E. \& Ford, J. M. Synergistic chemosensitivity of triple-negative breast cancer cell lines to poly(ADP-Ribose) polymerase inhibition, gemcitabine, and cisplatin. Cancer Res. 70, 7970-7980 (2010).

32. Ellard, S. L. et al. Randomized phase II study comparing two schedules of everolimus in patients with recurrent/metastatic breast cancer: NCIC Clinical Trials Group IND.163. J. Clin. Oncol. 27, 4536-4541 (2009).

33. Tryfonopoulos, D. et al. Src: a potential target for the treatment of triplenegative breast cancer. Ann. Oncol. 22, 2234-2240 (2011).

34. Augoff, K., McCue, B., Plow, E. F. \& Sossey-Alaoui, K. miR-31 and its host gene InCRNA LOC554202 are regulated by promoter hypermethylation in triplenegative breast cancer. Mol. Cancer 11, 5 (2012).

35. Li, S. et al. Long noncoding RNA GAS5 suppresses triple negative breast cancer progression through inhibition of proliferation and invasion by competitively binding miR-196a-5p. Biomed. Pharmacother. 104, 451-457 (2018).

36. Selbach, M. et al. Widespread changes in protein synthesis induced by microRNAs. Nature 455, 58-63 (2008).

37. Chen, Y. et al. miRNA-200c increases the sensitivity of breast cancer cells to doxorubicin through the suppression of E-cadherin-mediated PTEN/Akt signaling. Mol. Med. Rep. 7, 1579-1584 (2013).

38. Valastyan, S. et al. A pleiotropically acting microRNA, miR-31, inhibits breast cancer metastasis. Cell 137, 1032-1046 (2009).

39. Wang, C., Zheng, X., Shen, C. \& Shi, Y. MicroRNA-203 suppresses cell proliferation and migration by targeting BIRC5 and LASP1 in human triplenegative breast cancer cells. J. Exp. Clin. Cancer Res. 31, 58 (2012)

40. Okita, Y., et al. The transcription factor MAFK induces EMT and malignant progression of triple-negative breast cancer cells through its target GPNMB. Sci. Signal 10, 474 (2017).

41. Tang, J. et al. Molecular mechanisms of microRNAs in regulating epithelialmesenchymal transitions in human cancers. Cancer Lett. 371, 301-313 (2016). 
42. Song, S. J. et al. MicroRNA-antagonism regulates breast cancer stemness and metastasis via TET-family-dependent chromatin remodeling. Cell 154, 311-324 (2013).

43. Ranganathan, P., Weaver, K. L. \& Capobianco, A. J. Notch signalling in solid tumours: a little bit of everything but not all the time. Nat. Rev. Cancer 11, 338-351 (2011).

44. Louvi, A. \& Artavanis-Tsakonas, S. Notch and disease: a growing field. Semin. Cell Dev. Biol. 23, 473-480 (2012).

45. Wu, C. X. et al. Notch inhibitor PF-03084014 inhibits hepatocellular carcinoma growth and metastasis via suppression of cancer stemness due to reduced activation of Notch1-Stat3. Mol. Cancer Ther. 16, 1531-1543 (2017).

46. Takebe, N. et al. Targeting Notch, Hedgehog, and wnt pathways in cancer stem cells: clinical update. Nat. Rev. Clin. Oncol. 12, 445-464 (2015).
47. Jin, L., Vu, T., Yuan, G. \& Datta, P. K. STRAP promotes stemness of human colorectal cancer via epigenetic regulation of the NOTCH Pathway. Cancer Res. 77, 5464-5478 (2017)

48. Qiu, M. et al. Specific inhibition of Notch1 signaling enhances the antitumor efficacy of chemotherapy in triple negative breast cancer through reduction of cancer stem cells. Cancer Lett. 328, 261-270 (2013).

49. Clementz, A. G., Rogowski, A., Pandya, K., Miele, L. \& Osipo, C. NOTCH-1 and $\mathrm{NOTCH}-4$ are novel gene targets of PEA3 in breast cancer: novel therapeutic implications. Breast Cancer Res. 13, R63 (2011).

50. Pan, H. et al. Genistein inhibits MDA-MB-231 triple-negative breast cancer cell growth by inhibiting NF-kappaB activity via the Notch-1 pathway. Int J. Mol. Med 30, 337-343 (2012).

51. Zhou, W. et al. Upregulation of S100A16 expression promotes epithelialmesenchymal transition via Notch1 pathway in breast cancer. J. Biomed. Sci. 21, 97 (2014) 